\title{
Utilization of Near IR Absorbing Gold Nanocolloids by Green Synthesis Beste ELVEREN ${ }^{1, a}$, Ümit Hakan YILDIZ1,b and Ahu ARSLAN YILDIZ ${ }^{1, c}$ \\ ${ }^{1}$ İzmir Institute of Technology, Gülbahçe, İzmir, Turkey \\ abesteelveren@iyte.edu.tr, bhakanyildiz@iyte.edu.tr, ’ahuarslan@iyte.edu.tr
}

Keywords: green synthesis, gold nanoparticle, biofriendly, flavonoids, sage, plant extract

\begin{abstract}
The rapid developments in nanoscience, and its applications on biomedical areas have a large impact on drug delivery, tissue engineering, sensing, and diagnosis. Gold is widely investigated nanomaterial for the last couple of decades, since it has unique surface properties and very low toxicity to biological environment. In this work, we present a novel synthesis of gold nanoparticles (GNPs) exhibiting both visible and near-IR absorbance without agglomeration. The surface of GNPs were analyzed by routine methods and the binding kinetics were investigated by Surface Plasmon Resonance (SPR) Spectroscopy. The unique optical properties of near-IR asorbing GNP colloids hold promise for biological applications.
\end{abstract}

\section{Introduction}

The utilization of gold nanoparticles is an area of interest due to their plasmonic, and chemical properties, stability and biocompability [1-3]. There are physical and chemical protocols that are available for synthesizing GNPs. Plasma arching, ball milling, thermal evaporation, spray pyrolysis, ultra thin films, pulsed laser desorption, lithographic tecniques,sputter deposition, layer by layer growth, molecular beam epistaxis and diffusion flame synthesis are some of the physical techniques [4]. Chemical techniques include electrodeposition, sol-gel process, chemical solution, and vapour deposition [5, 6], Langmuir Blodgett technique, catalytic route, hydrolysis [7], co-precipitaiton, and wet chemical synthesis [8]. Physical and chemical techniques for synthesizing GNPs have been using strong reducing and stabilizing agents which leave harmful side products to the environment as well as has harmful side effects on human health.

Green synthesis emerges an environmental friendly technique of synthesizing nanoparticles. The green synthesis process utilizes natural and biomimetic materials such as: plant extracts, bacteria, algae and enzymes [9] as reducing agent. Different parts of plant like: root, stem, leaf, fruit, seed, callus, peel, and flower can be used for the synthesis [10]. For instance, Mukherjee et al. reported a green approach for synthesis of GNPs by using green tea derivatives [11]. In another study, green synthesis was done by using different fruit juices [12]. Similarly, poly-phenolic components of tea extracts were used for synthesizing nanoclusters which was investigated to inhibit cell proliferation and to induce apoptosis of cancer cells [13].

The use of phenolic content of plants for GNP synthesis provide kinetic control of metallization reaction, which affects the growth of nanoparticle [14].In this study, Salvia officinalis (common sage) extract was used as reducing agent for green synthesis. Phenolic components of Salvia officinalis such as: caffeic acid, vanillic acid and rosmarinic acid [15] have the active role on reduction reaction. The phenolic content of Salvia officinalis has a potential to exhibit profound effect on morphological, optical, and chemical properties resulting nanoparticles as represented in Fig. 1. In particular, electromagnetic radiation absorbance in near-IR region $(750-1500 \mathrm{~nm})$ has enhanced by phenolic capping of green synthesized GNPs. This unique optical characteristic of GNPs holds great promise in optical detection of biological species such as proteins. 


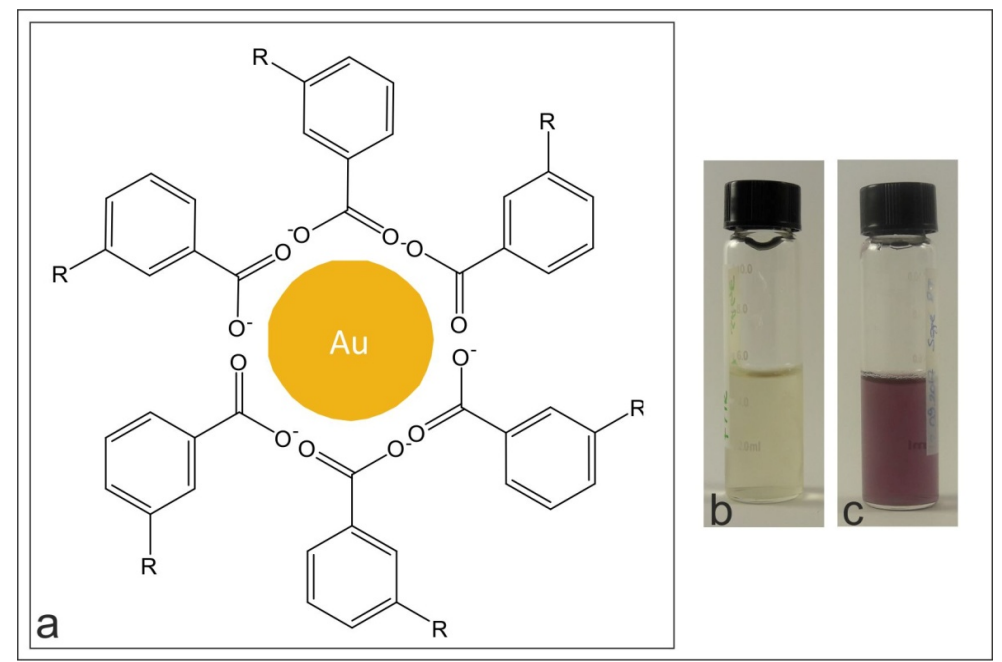

Fig. 1 Predicted structure and capping of GNPs; R groups represents phenolic compounds (a), gold solution (b), green synthesized GNP solution (c)

\section{Materials and Methods}

Chemicals. Gold(III) chloride hydrate (99.999\% trace metal basis) and poly-L-lysine hydrobromide (PLL, $0.05 \mathrm{mg} / \mathrm{mL}$ ) (MW 70.000 - 150.000) was purchased from Sigma-Aldrich Company (St. Louis, MO, USA). Ultrapure water (Sartorius Water Purification Systems - Arium Pro Ultrapure Water System) was used throughout the experiment. Dry sage leaves were purchased from local market.

Preparation of Sage Extracts. $4.0 \mathrm{~g}$ of dry sage leaves were washed once with tap water then washed with ultrapure water (UPW). The leaves were diced in a blender with $30 \mathrm{~mL}$ UPW until a homogeneous liquid was obtained. Then the volume was topped to $50 \mathrm{~mL}$ with UPW. The obtained liquid was first filtered through $120 \mathrm{~mm}$ filter paper, then $0.2 \mu \mathrm{m}$ filter paper to remove any solid particle. The extracts then stored at $-20^{\circ} \mathrm{C}$ up to 10 months until further analysis.

Gold(III) chloride hydrate Solution Preparation. $0.01 \mathrm{~g}$ of solid $\mathrm{HAuCl}_{4}$ dissolved in $100 \mathrm{~mL}$ UPW to get final concentration of $0.1 \mathrm{~g} / \mathrm{L}$. The solution stored at $4^{\circ} \mathrm{C}$.

GNP Sample Preparation. $375 \mu \mathrm{L}$ of sage extract was mixed with $5 \mathrm{~mL}$ of gold solution in glass vials. The characterizations were done at varied temperatures $\left(4^{\circ} \mathrm{C}, \mathrm{RT}\right)$ and time scales $(2$ hours, 1 day, 2,3 and 4 days) to characterize the GNP formation and reaction length.

Spectrophotometric Analysis. The absorbance response of both the extract and nanoparticle solutions was measured with Thermo Scientific Multiscan GO Microplate Reader (Thermo-Fisher Scientific Inc.,MA,USA). Absorbance analysis was used periodically to qualify and quantify the reaction efficiency.

GNP Size Measurements. Malvern Zetasizer Nano ZS (Malvern Instruments,UK) device was used to measure the size of GNPs. The analysis was done immediately after synthesis, and weekly. Samples were analyzed prior to synthesis without further treatment.

Atomic Force Microscopy Analysis. Atomic Force Microscopy (AFM) measurements for topographical analysis was undertaken with Multimode 8-HR AFM (Bruker Corp., MA). AFM samples are prepared by using $1 \times 1 \mathrm{~cm}$ PMMA blocks covered with aluminum foil, and then $100 \mu \mathrm{L}$ PLL was incubated for 24 hours. After washing twice, $100 \mu \mathrm{L}$ gold nanoparticle solution was incubated 24 hours which was then washed twice and proceeded with analysis..

Fourier Transform Infra Red Spectroscopy Analysis. Fourier Transform Infra Red Spectroscopy analysis was done by Perkin Elmer Spectrum 100 FT-IR Spectrometer (Perkin Elmer Inc., USA). Both the extract and GNP solution was freezed at $-20^{\circ} \mathrm{C}$ and then lyophilized over night to obtain 
powder form. $\mathrm{KBr}$ pellet was used as a control over the extract and GNP solid was mixed with $\mathrm{KBr}$ pellets to measure ther FT-IR spectra.

Surface Plasmon Resonance Spectroscopy Analysis. Binding mechanisms of GNPs to the gold slides (47 nm thick gold surface) were investigated by SPR setup (Nanodev Scientific Inc.,TR). The gold slides were cleaned with isopropyl alcohol (IPA) for $15 \mathrm{~min}$, then the slide was put into ethyl alcohol for further cleaning. After cleaning, both sides of the surface dried with $\mathrm{N}_{2}$ gas to remove any moisture. For analysis, $600 \mu \mathrm{L}, 0.05 \mathrm{mg} / \mathrm{mL}$ PLL was incubated for 24 hours, then GNP colloid solution was incubated for 24 hours. SPR measurement was taken in kinetic mode to observe the GNP binding.

\section{Results and Discussion}

Spectrophotometric Analysis. As shown in Fig. 2, green synthesized GNPs have specific absorbance at 536 and $980 \mathrm{~nm}$ with an intensity of 0.23 and 0.12 in optical density. The trials for the reaction period varying from 24 to 96 hours showed no significant difference in absorbance spectra which illustrates metallization has been kinetically controlled at 24 hours. Absorbance peak at $536 \mathrm{~nm}$ corresponds to typical colloidal GNPs with a size of 50 to $60 \mathrm{~nm}$ [16]. A broad peak appeared from 750 to $1000 \mathrm{~nm}$ with a maximum at $950 \mathrm{~nm}$ was found to be a characteristic. The near IR property arises due to the controlled self-assembly of gold colloids by the phenolic moieties

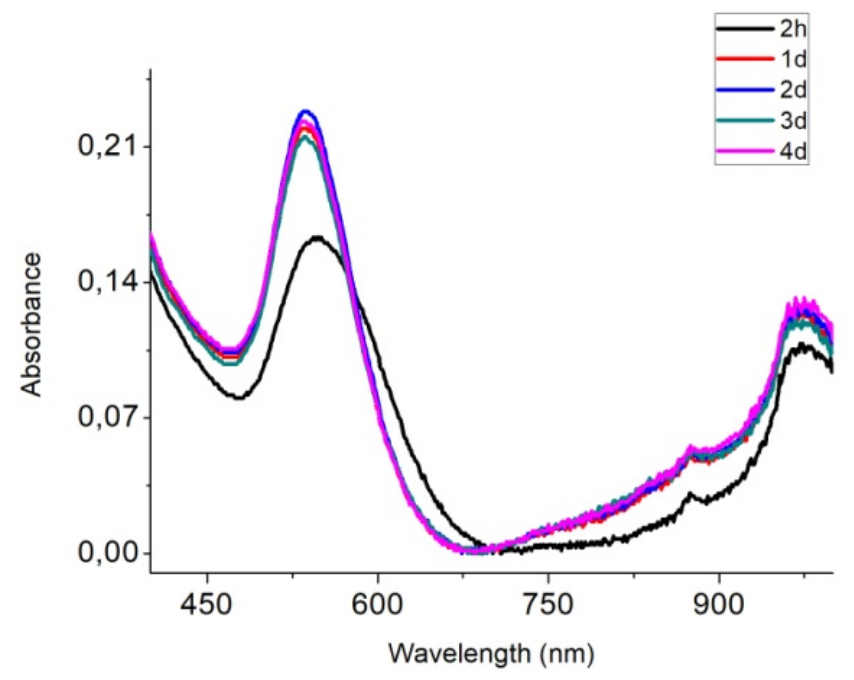

Fig. 2 Absorbance spectra of GNPs varying from 2 hours to 4 days

Size Measurements. The size of GNPs during growth reaction and also post reaction has been given in Fig. 3. In 2 hours GNPs reach to $56.3 \mathrm{~nm}$ size in diameter and by completion of reaction there is no significant change in size. These results also confirm that optical characterization results presented in Fig. 2; no substantial variation in size by the completion of reaction. To monitor clustering, aggregation and colloidal stability of GNPs, three week stabilty test has been conducted. As presented in Fig. 3, size of the GNPs changed maximum $10 \mathrm{~nm}$ after three weeks that assures colloids exhibit fair stability as compare ro near-IR absorbing nano structures such as nanorods. 


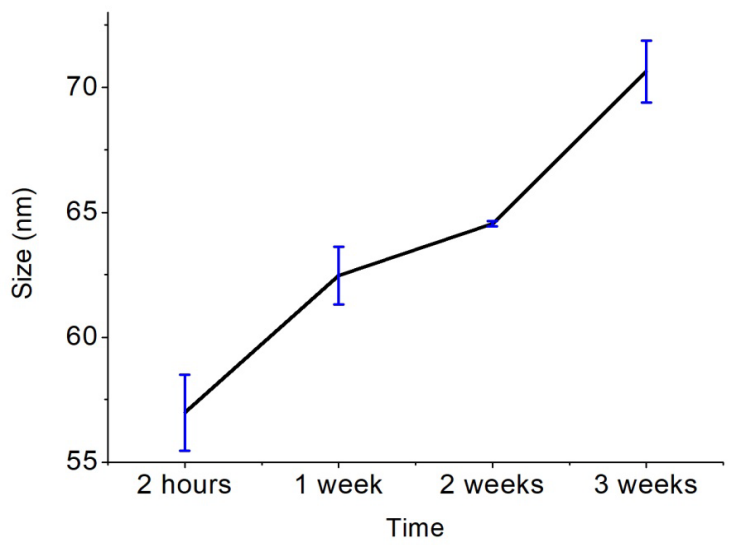

Fig. 3 Size measurements of GNPs varying from 2 hours to 3 weeks

AFM Analysis. Binding of GNPs and the even distribution on solid surface is confirmed by the AFM results given in Fig. 4. Similary, 3D images supports the even distribution. The periodic structure of GNPs on flat gold surface has large impact for plasmonic sensing applications.

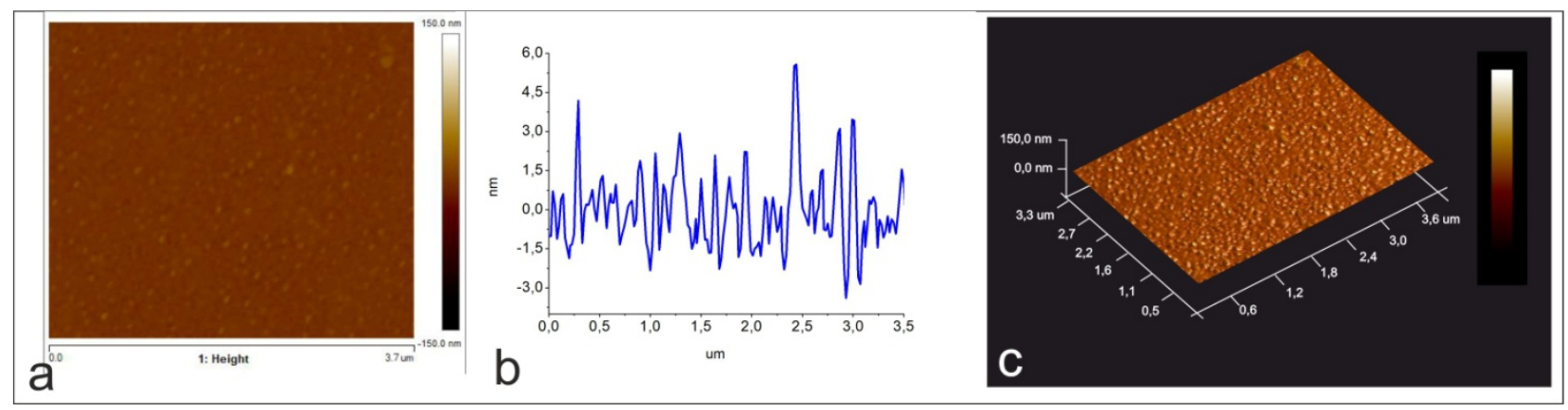

Fig. 4 AFM images of GNPs on solid surface; 2D images of surface (a) surface profile (b), and 3D images of surface (c)

FTIR Analysis. A FTIR spectra of Salvia officinalis is given in Fig.5. Both sage extract and GNP solution has $\mathrm{O}-\mathrm{H}$ stretching at 3404.32 and 3423.19 respectively. Also, between $3300-3500 \mathrm{~cm}^{-1}$ the peak can be related to $2^{\circ}$ amine groups. Generally, between $3000-2800 \mathrm{~cm}^{-1}$, we observe two peaks that is specific to self assembly, which are at 2850 and $2918 \mathrm{~cm}^{-1}$. A finger print signal was observed at $2850.65,2867.99,2930.97 \mathrm{~cm}^{-1}$, which is an indication of self assembled structures and capping around GNPs (Fig.6). Also, the signal around $1700 \mathrm{~cm}^{-1}$ shows that both samples have carbonyl groups. At 1610.71 and $1632.79, \mathrm{~N}-\mathrm{H}$ bending was observed for both sage extract and GNP solution, which supports amine group existence. The only difference in the spectrum between sage extract and GNP solution is the sage extract peak at $1407.52 \mathrm{~cm}^{-1}$ that dissappears from the GNP spectra. This peak shows the nitro $\left(\mathrm{NO}_{2}\right)$ compounds stretching. At $1268 \mathrm{~cm}^{-1}$, we observe phenolic acyl groups. The peaks at 1053.10 and $1108.20 \mathrm{~cm}^{-1}$ represent the alcoxy $(\mathrm{C}-\mathrm{O})$ stretching signals. Also at 618.04 and $656.10 \mathrm{~cm}^{-1}$, the same characteristic peaks was obtained for aromatic $\mathrm{sp}^{2}$ $\mathrm{C}-\mathrm{H}$ bending. 


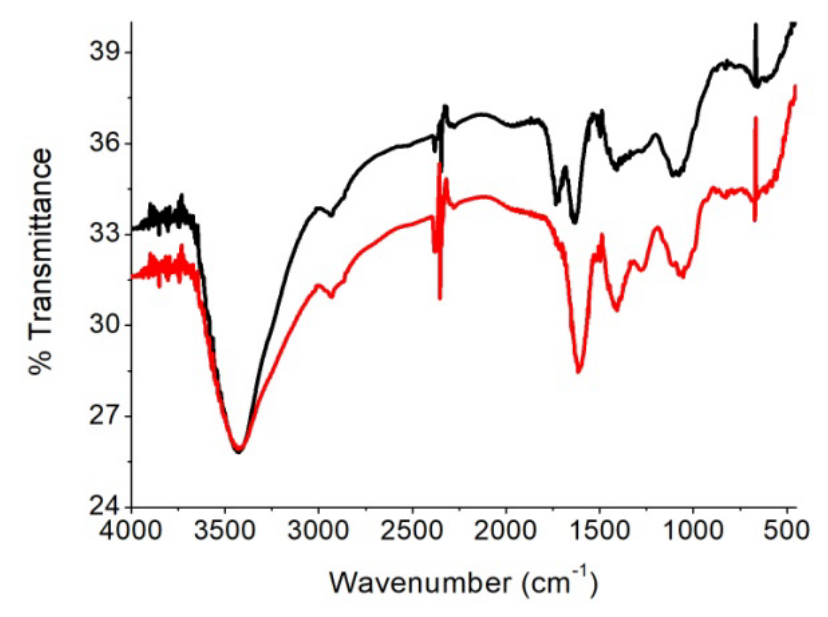

Fig. 5 FTIR spectrum of sage extract (red), and Au GNP (black)

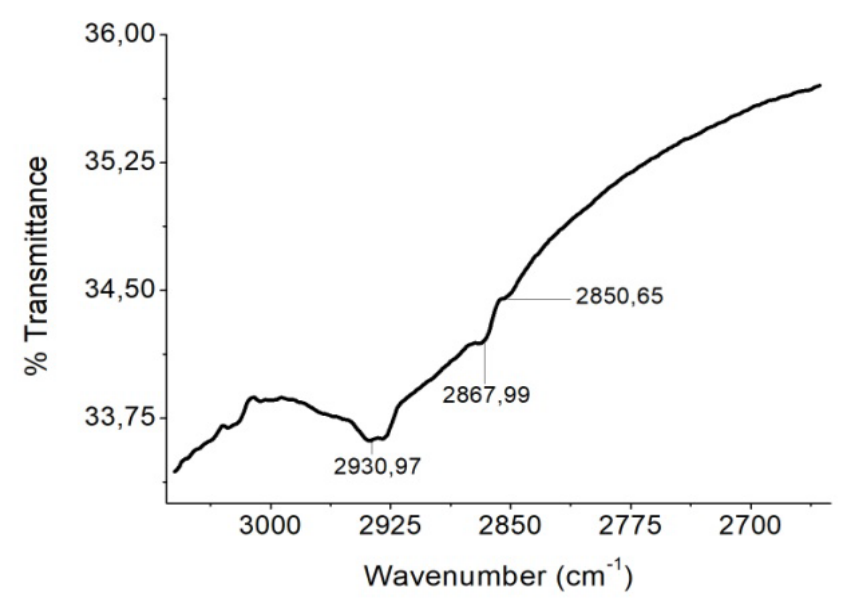

Fig. 6 FTIR spectrum of self assembly and capping around GNP

SPR Spectroscopy Analysis. Fig. 7 shows the SPR binding kinetics of both citrate capped and phenolic capped GNPs. In this analysis, a known and well characterized citrate capped GNPs were used to compare our method with the traditional strong reducing agent; citric acid. Citrate capping on GNPs are one of the most used technique for metallization of gold, and it is being used in many applications as mentioned above [17-20]. Both solutions reach their maximum adsorbtion value after one hour. From Fig.7, it can be interpretted that GNPs synthesized by phenolic reduction has a good binding ability, and binding efficiency is also comparable to the traditional citrate capped GNPs.

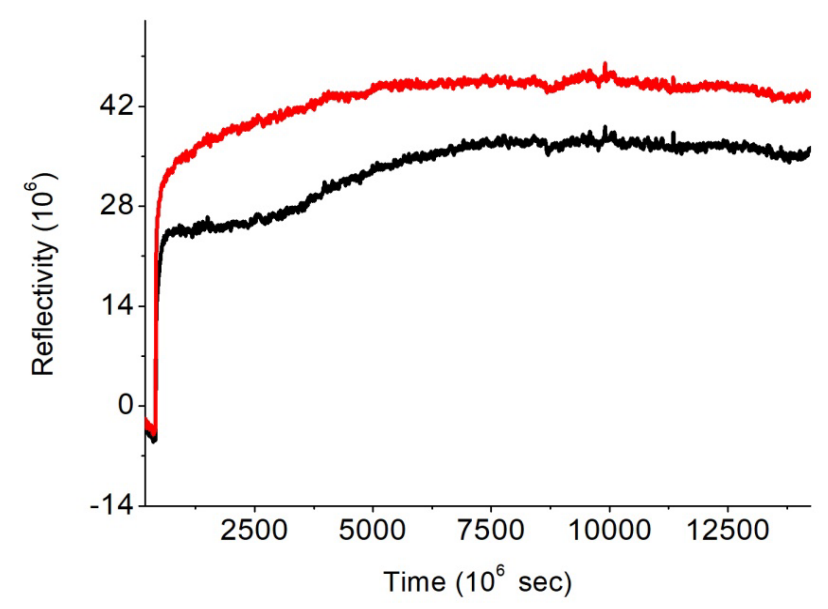

Fig. 7 SPR Spectrum of citrate capped (black) and phenolic capped (red) GNPs 


\section{Conclusion}

The biological applications of GNPs strongly depends on how much information can be obtained from the GNPs and their surface. Body response starts from the surface, which is why surface functionalization and characteristic of GNPs is an important parameter for biological applications. The characterizations show that the GNPs synthesized with common sage extract has stable diameter over time. On the surface of the GNPs, a self assembly structure was observed and the phenolic groups in the extract cause this self-assembly. Apart from the classical approaches of synthesizing GNPs, such as citrate reduction, our green synthesis method proves that more biofriendly GNP synthesis is possible and biological applications can be carried outwithout the harmful effects of side-products.

\section{Acknowledgements}

Authors want to thank to İzmir Institute of Technology Scientific Research Project (BAP) 2016 IYYTE70.

\section{References}

[1] A.J. Mieszawska, W.J. Mulder, Z.A. Fayad, D.P. Cormode, Multifunctional gold nanoparticles for diagnosis and therapy of disease, Mol Pharm 10(3) (2013) 831-47.

[2] M.C. Daniel, Astruc, D., Gold Nanoparticles: Assembly, Supramolecular Chemistry, QuantumSize-Related Properties, and Applications toward Biology,Catalysis, and Nanotechnology, Chemical Reviews 104 (2004) 293-346.

[3] A.S. Thakor, J. Jokerst, C. Zavaleta, T.F. Massoud, S.S. Gambhir, Gold nanoparticles: a revival in precious metal administration to patients, Nano Lett 11(10) (2011) 4029-36.

[4] R. Joerger, Klaus, T., Granqvist, C. G., Biologically Produced Silver-Carbon Composite Materials for Optically Functional Thin-Film Coatings, Advanced Materials 12(6) (2000) 407-409.

[5] S. Panigrahi, S. Kundu, S. Ghosh, S. Nath, T. Pal, General method of synthesis for metal nanoparticles, Journal of Nanoparticle Research 6(4) (2004) 411-414.

[6] M.M. Oliveira, D. Ugarte, D. Zanchet, A.J.G. Zarbin, Influence of synthetic parameters on the size, structure, and stability of dodecanethiol-stabilized silver nanoparticles, Journal of Colloid and Interface Science 292(2) (2005) 429-435.

[7] M.P. Pileni, Nanosized Particles Made in Colloidal Assemblies, Langmuir 13(13) (1997) 32663276.

[8] P.P. Gan, S.H. Ng, Y. Huang, S.F.Y. Li, Green synthesis of gold nanoparticles using palm oil mill effluent (POME): A low-cost and eco-friendly viable approach, Bioresource Technology 113 (2012) 132-135.

[9] S. Iravani, Green synthesis of metal nanoparticles using plants, Green Chemistry 13(10) (2011) 2638-2650.

[10] P. Kuppusamy, M.M. Yusoff, G.P. Maniam, N. Govindan, Biosynthesis of metallic nanoparticles using plant derivatives and their new avenues in pharmacological applications - An updated report, Saudi Pharm J 24(4) (2016) 473-84.

[11] S. Mukherjee, S. Ghosh, D.K. Das, P. Chakraborty, S. Choudhury, P. Gupta, A. Adhikary, S. Dey, S. Chattopadhyay, Gold-conjugated green tea nanoparticles for enhanced anti-tumor activities and hepatoprotection--synthesis, characterization and in vitro evaluation, J Nutr Biochem 26(11) (2015) 1283-97. 
[12] J. Chou, X. Li, Y. Yin, N. Indrisek, Determination of antioxidant activities in fruit juices based on rapid colorimetric measurement and characterisation of gold nanoparticles, International Journal of Environmental Analytical Chemistry 95(6) (2015) 531-541.

[13] A.A. Alshatwi, J. Athinarayanan, V.S. Periasamy, Green synthesis of bimetallic Au@Pt nanostructures and their application for proliferation inhibition and apoptosis induction in human cervical cancer cell, J Mater Sci Mater Med 26(3) (2015) 148.

[14] P. Elia, R. Zach, S. Hazan, S. Kolusheva, Z. Porat, Y. Zeiri, Green synthesis of gold nanoparticles using plant extracts as reducing agents, Int J Nanomedicine 9 (2014) 4007-21.

[15] W. Zheng, Wang, S.Y., Antioxidant Activity and Phenolic Compounds in Selected Herbs, J. Agric. Food Chem 49 (2001) 5165-5170.

[16] J. C. Martínez, N. A. Chequer, J. L. González, T. Cordova, Alternative Metodology for Gold Nanoparticles Diameter Characterization Using PCA Technique and UV-VIS Spectrophotometry, Nanoscience and Nanotechnology 2(6) (2013) 184-189.

[17] D. Curry, A. Cameron, B. MacDonald, C. Nganou, H. Scheller, J. Marsh, S. Beale, M.S. Lu, Z. Shan, R. Kaliaperumal, H.P. Xu, M. Servos, C. Bennett, S. MacQuarrie, K.D. Oakes, M. Mkandawire, X. Zhang, Adsorption of doxorubicin on citrate-capped gold nanoparticles: insights into engineering potent chemotherapeutic delivery systems, Nanoscale 7(46) (2015) 19611-19619.

[18] N. Pernodet, X.H. Fang, Y. Sun, A. Bakhtina, A. Ramakrishnan, J. Sokolov, A. Ulman, M. Rafailovich, Adverse effects of citrate/gold nanoparticles on human dermal fibroblasts, Small 2(6) (2006) 766-773.

[19] S.J. Corr, M. Raoof, Y. Mackeyev, S. Phounsavath, M.A. Cheney, B.T. Cisneros, M. Shur, M. Gozin, P.J. McNally, L.J. Wilson, S.A. Curley, Citrate-Capped Gold Nanoparticle Electrophoretic Heat Production in Response to a Time-Varying Radio-Frequency Electric Field, J Phys Chem C $116(45)$ (2012) 24380-24389.

[20] J.F.A. de Oliveira, M.B. Cardoso, Partial Aggregation of Silver Nanoparticles Induced by Capping and Reducing Agents Competition, Langmuir 30(17) (2014) 4879-4886. 